\title{
A New Image Quality Index for Objectively Evaluating Despeckling Filtering in SAR Images
}

\author{
Luis Gomez, Member, IEEE, Maria Elena Buemi, Julio C. Jacobo-Berlles, and Marta E. Mejail
}

\begin{abstract}
Synthetic aperture radar (SAR) images are corrupted with a multiplicative granular-like noise pattern known as speckle. The goal for a despeckling filter consists of suppressing the speckle while preserving all the scene features such as texture, point-type targets, and, especially, edges. There exist several speckle filtering techniques and a relevant number of image quality indexes to evaluate the performances of a filtering operation on an SAR image. However, assessing the superiority of a filter over other is not a trivial issue. In this work, we present a new referenceless estimator ( $\alpha \beta$-ratio estimator) based on the ratio edge detector which allows helping in objectively evaluating a filter realization on SAR images. The proposed estimator operates on the ratio image obtained as the point-to-point ratio between the original image (noisy image) and the filtered image. An ideal filter operation on an image implies that, in areas where speckle is fully developed, the ratio image should have the features of pure speckle and no geometric content. The new estimator measures the remaining geometric content within the ratio image. This new estimator is easy to compute and it provides an excellent metric to rank a filtering operation on real SAR images.
\end{abstract}

Index Terms-Image-quality index, ratio images, speckle, speckle filtering, synthetic aperture radar (SAR).

\section{INTRODUCTION}

$\mathbf{S}$ AR SYSTEMS generate images by coherent processing of the scattered signals and consequently, they are susceptible to speckle noise [1]. Speckle is not truly a noise in the signal processing sense and it indeed provides useful information. However, speckle makes SAR images more difficult to interpret and filtering is required to better improve postprocessing steps such as image segmentation or image classification [2].

Speckle filtering for SAR images is a very active area of research since the years when some of the most known filters were low-pass averaging filters, filters based on underlying image statistics [3], and diffusion-like filters [4]. New approaches based on the nonlocal-means (NL-means) filters minimizing a Bayesian risk to reduce white Gaussian noise have appeared recently [5] and from that, their natural extension are known as Bayesian NL-means filters [6] which aim to reduce the Bayesian risk by estimating the prior probability on the noisy image patch.

However, as it is well accepted, there is no ideal despeckling filtering. To establish the superiority of any despeckling filter

Manuscript received October 30, 2014; revised June 02, 2015; accepted July 29, 2015. Date of publication August 17, 2015; date of current version February 22, 2016.

L. Gomez is with the Department of Electronic Engineering and Automatic (DIEA), University of Las Palmas de Gran Canaria, Las Palmas de Gran Canaria 35017, Spain (e-mail: luis.gomez@ulpgc.es).

M. E. Buemi, J. Jacobo-Berlles and M. E. Mejail are with Computation and Image Processing Group, University of Buenos Aires, Buenos Aires, Argentina (e-mail: mebuemi@dc.uba.arg; julio@dc.uba.arg; marta@dc.uba.arg).

Digital Object Identifier 10.1109/JSTARS.2015.2465167 over others is not a trivial issue even for those cases where a ground-truth image (commonly a synthetic image) to compare results with is available. Sometimes, there is no special interest to obtain the optimal filtered image. In [7], the user guides the design of an NL-means Bayesian filter to get not an optimal design but his desired design, i.e., a subjective solution satisfying the user's criteria.

Despeckled images are usually evaluated using some image quality index. A large body of literature exists regarding such indexes. Among them, the peak signal-to-noise ratio (PSNR), the $\beta$ correlator [8], Pratt's figure of merit $(F O M)$ [10], and the structural similarity (SSIM) index [11] are well established and commonly used by researches within the SAR community. To correctly evaluate an image quality index, a ground-truth image (noiseless) is required, and for most real cases (real SAR images), such a ground-truth image is not available.

For cases where this is not possible, the estimations of image mean preservation and variance reduction (both estimated in a user-selected homogeneous area) are mandatory. Other researchers use the combination of those simple statistical quality-indexes to define the equivalent number of looks (ENLs), which must be as high as possible for the denoised image. For a given image $I$ and a chosen homogeneous region of interest (ROI) with a mean value $\mu$ and a standard deviation $\sigma$, the $E N L$ is estimated by $\mu^{2} / \sigma^{2}$. Larger $E N L$ values indicate stronger speckle rejection, i.e., an improved ability to tell apart the different gray levels within the image. A new estimator, blind/referenceless image spatial quality evaluator (BRISQUE) [12] has recently appeared to overcome the problem of evaluating a filtered image without needing a ground-truth reference image. This estimator is intended for additive Gaussian noise.

In this paper, we deal with real images when no ground-truth image is available to assess the performance of a specklefiltering operation on real SAR images.

We propose a new referenceless image estimator for real SAR images. The proposed estimator operates on the ratio image obtained as the point-to-point ratio between the original image (noisy image) and the filtered image. The use of ratio images to evaluate a filtering operation is common in recent publications [5], [13], [7]. This is also becoming a standard analysis in the SAR community and it consists of visually inspecting the regularity pattern shown in the ratio image and estimating the $E N L$ in a homogeneous area within this ratio image. An ideal filter operation on an image implies that, in areas where speckle is fully developed, this ratio should show the features of pure speckle [8]. The ENL estimated should approach to the ENL of the original SAR image. According to the multiplicative model [9], the mean of the speckle is 1 . 
Therefore, for an ideal filtering, the mean of the ratio image should also be 1 .

Our main goal in this work is to provide an efficient estimator to objectively evaluate a filtering operation in SAR images. We remark that the new estimator can be used exclusively to better design a given despeckling filter through the ranking of multiple objectively evaluations spanning into filter design parameters. We present some results dealing with this important application of the proposed estimator. Besides, although we do not focus on comparing well-established despeckling filters, it is tempting to apply the new estimator to rank a filtering operation on real SAR images. Some promising results pursuing this goal are also shown.

Although not explored in this paper, the proposed referenceless estimator may also be embedded into the design of the filter, taking advantage of the objectively evaluation it provides, to enhance the capabilites of a despeckling filter.

This paper is organized as follows. In Section II, some filtering results for a real SAR image are presented to fully justify this work. Section III deals with the standard ratio edge detector for detecting edges in SAR images and its adaption to detect edges in ratio images. The new image-quality index, the $\alpha \beta$-ratio estimator, is also discussed in this section. Section IV shows some results for real SAR images, and finally, in Section V, some conclusion is drawn.

\section{Some Results for COMmonly Applied DESPECKLING FILTERS}

In this section, some examples using well-established SAR despeckling filters are provided to justify the need of a new referenceless image quality estimator to evaluate a filtering operation on an SAR image.

To point out the need of a new referenceless image quality index for SAR images, we show in Fig. 1 some results for the real SAR intensity image (top left) corresponding to a ROI $(500 \times 500$ samples $)$ of the 1-look HH SAR (Oberpfaffenhofen, Germany). This image contains clear-cut areas, bright scatterers, urban areas with geometric content (buildings, roads), and homogeneous areas. As seen, the chosen ROI for the quantitative test corresponds to a forest area.

The results using the original Lee filter [14] (top right) and the speckle reducing anisotropic diffusion (SRAD) filter for two filtering designs (bottom left and bottom right, respectively) are also shown in Fig. 1.

The Lee filter is an adaptive filter specially designed to eliminate speckle noise while preserving edges and point features (like strong reflectors) in SAR images. This filter uses a linear speckle noise model and its purpose is to reduce speckle in terms of minimizing the mean square error. The filter is adaptive in the sense that it proceeds by estimating locally the image mean and the image variance within a small region (usually a $3 \times 3$ or $7 \times 7$ mask) and combining this information with the $E N L$ of the image, which is an input parameter to the filter. From that, the filter operates as an identity filter in edges, which results in little modification to the pixel values near edges. In homogeneous areas, the filter leads to the same result as that of the mean filter.

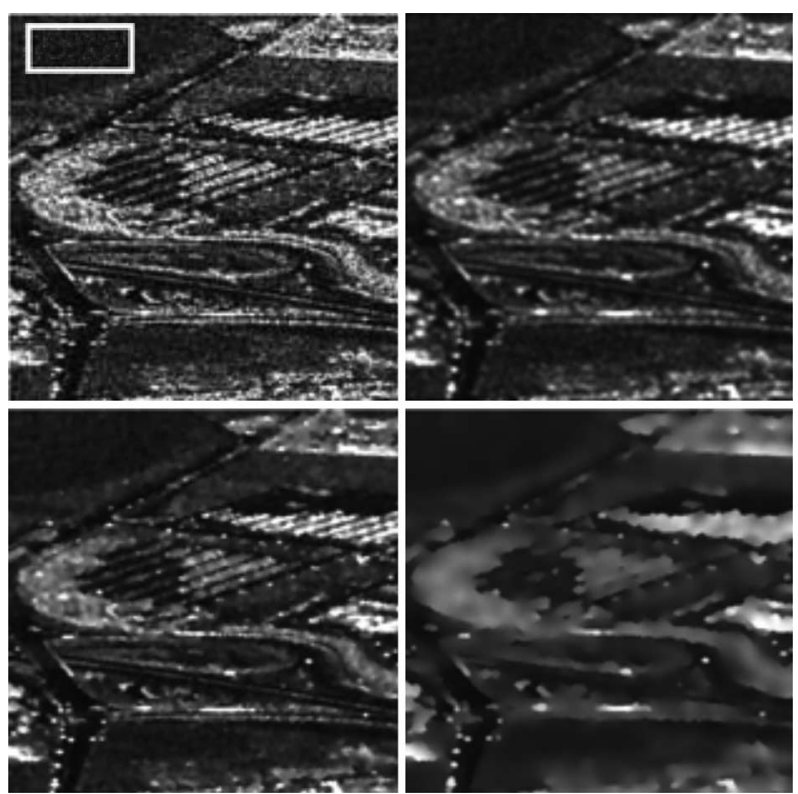

Fig. 1. Real SAR image (top left). (Top right) Lee filter (mask size: $7 \times 7$ ), the SRAD filter ( $t=15$, bottom left), and overfiltered SRAD filter $(t=100$, bottom right).

The SRAD filter can be seen as a mixture of the classical anisotropic diffusion filter [15] and the adaptive speckle Lee filter. The advantages of anisotropic diffusion include intraregion smoothing and edge preservation, performing well for images corrupted by additive Gaussian noise. However, anisotropic diffusion, instead of reducing speckle, will enhance it for those images containing such multiplicative noise. In [4], the authors propose an anisotropic diffusion version of the Lee filter showing the effectiveness of SRAD filter. SRAD filter better preserves and will enhance edges while efficiently removes speckle in homogeneous regions. As a notable difference from other filters, SRAD filter is formulated in continuous domain through a partial differential equation (PDE), which implies that no convolution windows (masks) are needed. Due to that, it operates simultaneously within the image and as a consequence of that, the related computational cost is significantly reduced.

The SRAD filter handles some input parameters: $t$, diffusion time (number of iterations) and a local statistical estimate of the speckle in a homogeneous area at each $t$, which it is conveniently avoided through the use of a function modeled by two parameters. From that, it is clear that the SRAD filter needs to be well designed to suit to images to be filtered. Fortunately, the tuning of SRAD filter is not a hard task.

As it can be seen from results shown in Fig. 1, the Lee filter and the SRAD filter $(t=15)$ perform well on despeckling the noisy image. Both filters preserve borders and the strong reflectors (which are of most importance on SAR filtering) are also kept. Quantitative results measured on the ROI shown in the noisy image (top left) for the mean, and $E N L$ estimators are provided in Table 1. Note that $E N L=\mu^{2} / \sigma^{2}$, so that standard deviation is also estimated (but not shown in Table I).

Note that the overfiltered result shown on the bottom right for the SRAD filter is visually far from the ideal solution (too 
TABLE I

QUANTITATIVE EVALUATION FOR THE REAL SAR IMAGE ESTIMATED IN THE SELECTED ROI (Best VALUES IN BoldFACE)

\begin{tabular}{|l||l|l|}
\hline Image & Mean & ENL \\
\hline \hline SAR image & 15.2997 & 0.9198 \\
\hline Lee filter $(7 \times 7)$ & $\mathbf{1 5 . 2 8 4 8}$ & 10.5888 \\
\hline SRAD filter $(t=15)$ & 15.3197 & 11.6270 \\
\hline SRAD filter $(t=100)$ & 14.9958 & $\mathbf{4 5 . 4 8 7 0}$ \\
\hline
\end{tabular}

much edge blurring). However, quantitative results (measured in the single ROI shown in the figure) proclaim the opposite (higher $E N L$ value and a reasonable mean preservation value). Additionally, visual result for the selected ROI is excellent. Therefore, it is not obvious how to select an ROI (or a number of ROIs) to get a mean preservation value and an ENL value consistent with visual results. This overfiltered result for the SRAD filter has been obtained using a larger diffusion time $(t=100)$ and also tuning the filter parameters to avoid the well-known salt and pepper noise related to these overfiltered cases for the SRAD filtering operation.

To decide whether the Lee filter has produced a better result than the SRAD filter is not an easy issue to answer. For cases having a ground-truth image to compare results with, numerical well-known estimators, such as FOM (Prattś FOM), SSIM index, PSNR and so on, may help to assess the outperforming of one filter over others. However, for real SAR images there is, generally, no ground-truth image to compare with, which makes more difficult to test a despeckling filter. Note that the availability of the ground-truth image (phantom) is reduced to academic study through filtering of synthetic images. Additionally, it is impossible to include all the reality expected in a real SAR image in a phantom, so filter performances must be decided upon filtering real SAR data.

In author's experience, given some results from several despeckling filters, there are SAR experts that prefer one solution over the other [7]. Additionally, one may ask what the purpose of such filtering is, because, one solution may be desired in terms of the required image postprocessing operation (image classification and edge segmentation) or in terms of demanding a lower computational time. Besides, values for some estimators may provide contradictory results (for instance, excellent FOM values but poor SSIM and PSNR values) which strongly complicates the analysis.

Additionally, for real SAR images, there is no ground-truth image to compare with and therefore, any filtering operation must be evaluated on the visual appearance (which is quite subjective) and on the numerical estimators provided by the $E N L$ and the mean preservation and variance reduction figures. It is this subjective evaluation for the visual inspection of filtered images what we try to avoid by applying the proposed ratio-based referenceless estimator.

\section{A. Visually Inspection of Ratio Images}

It is clear that no universal quality measure for filtered SAR images exists. The use of ratio images to evaluate a filtering operation is common in recent publications [5], [13], [7]. This

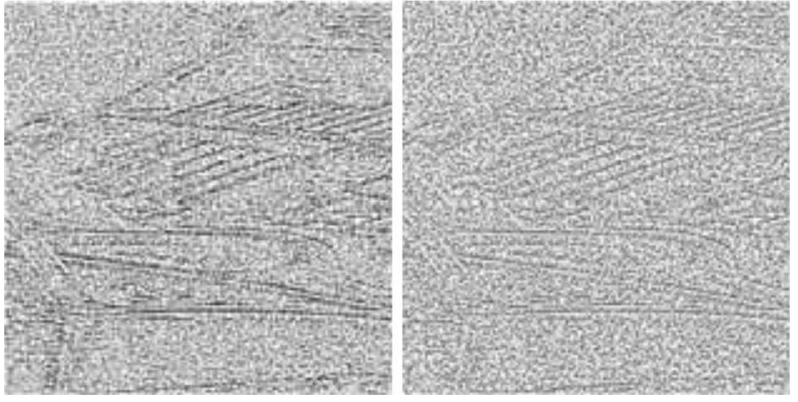

Fig. 2. Ratio images for the Lee filter with mask size $7 \times 7$ (left) and the SRAD $(t=15)$ filter (right).

is also becoming a standard analysis in the SAR community and it consists of inspecting the regularity pattern shown in the socalled aspect ratio image $\Pi=U / V$, where $U$ is the SAR original image and $V$ is its denoised version. An ideal filter operation on image $U$ implies that, in areas where speckle is fully developed, this ratio should show the features of pure speckle.

Additionally, for a reasonable despeckling operation, the presence in the ratio image $\Pi$ of geometric structures or any detail correlated with the original image $U$ indicates that some possible relevant information (for instance, edges or bright scatterers) have been removed or modified. Note that any content within the ratio image apart from pure speckle indicates that some modification regarding the nonhomogeneous areas (edges, mainly) has been performed by the filter. An ideal filter would not alter such edges or bright scatterers and therefore, ratio images would show a pure speckle pattern.

Therefore, following [8], for a noisy image (original SAR image) with $E N L=E N L_{\text {noisy }}$ (measured in a homogeneous area) and corrupted by speckle with mean value 1 , the best filter is the one for which the ratio image has the $E N L_{\text {ratio }}$ value closer to $E N L_{\text {noisy }}\left(E N L_{\text {noisy }} \approx E N L_{\text {ratio }}\right)$ and the mean value of the speckle measured in the ratio image $\mu_{\text {ratio }}$ closer to $1\left(\mu_{\text {ratio }} \approx 1\right)$.

In Fig. 2, the ratio images corresponding to the above filtered images using the Lee filter and the SRAD filter $(t=15)$ are illustrated. As it can be seen on these figures, clearly visible geometric structures still remain, which indicates that despeckling filtering (for both filters) has not been correctly performed. As mentioned above, only speckle should remain and should be the only information content of the filtered images.

Note that the geometric contents for both filters are comparable, although for the SRAD filter, the content appears notably less marked.

\section{Ratio Edge-Based Estimator for SAR Images}

From above, it is clear that some information is available within the ratio images apart from the pure visual one. In previous works, these images are either only evaluated visually, showing the ratio images and pointing out the remaining geometric content [7] or numerically by means of the evaluation of speckle statistics. For instance, in [8], the ENL values are measured in the ratio images and compared to the ideal expected ones. In [13], the ratio images are visually inspected and the 

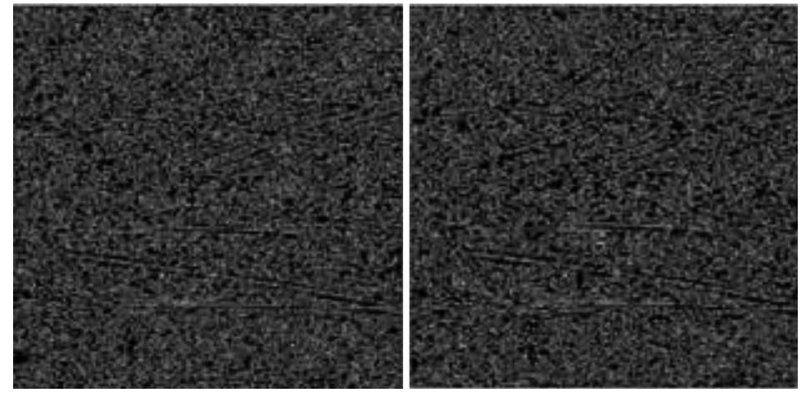

Fig. 3. Edges extracted by using Canny's detector for the ratio images for the Lee filter with mask size $7 \times 7$ (left) and the $\operatorname{SRAD}(t=15)$ filter (right).

expected value of intensity ratio is considered. But a sound analysis combining both estimates (visual and numerical) has not been done.

It seems natural to try to detect geometric content within the ratio image and to measure it in order to provide a quantitative figure. This is explained in Section III-A.

\section{A. Measuring the Geometric Content in Ratio Images}

To detect the amount of geometric content within the ratio images, i.e., to provide a numerical score for a given ratio image would be of maximum interest. In that manner, it will allow to objectively decide which filtering operation has better removed speckle from the original noisy image.

To extract the geometric content from the ratio image, several approaches were tested. Among them, statistical analysis of the ratio image under some probability density function (pdf) distributions, histogram analysis, and also texture analysis were explored. However, the results obtained were not convincing enough or easy-to-apply to implement a practical image quality index. Then, a first attempt relying on edges was applied and some initial results were obtained and then notably improved.

As a first approach, we can use any edge extractor, but, as it is shown in Fig. 3, the inherently full speckle-like content of the ratio images makes it hard, if not impossible, to apply it to extract valuable information. Note that Canny detector [16] handles some control parameters to get an optimal solution but, for ratio images and all the set of input parameters tested, no better results were obtained.

\section{B. Ratio Edge Detector}

To detect edges within the ratio images, the ratio edge detector from [17] is used. This edge detector performs well on SAR noisy images [17], [18], and it has been applied to this task for SAR images for years. Note that this edge detector has been applied to despeckled SAR images. We propose in this work to apply it to detect edges within the ratio images.

The ratio edge detector is defined as the ratio of the average (arithmetic average for a power image and quadratic average for an amplitude image) of the pixel values of two nonoverlapping neighborhoods on opposite sides of the points (see Fig. 4). That is, for a power image $I$, and two nonoverlapped regions $X_{1}$ and $X_{2}$ computed on the opposite side of the pixel $n$ under analysis
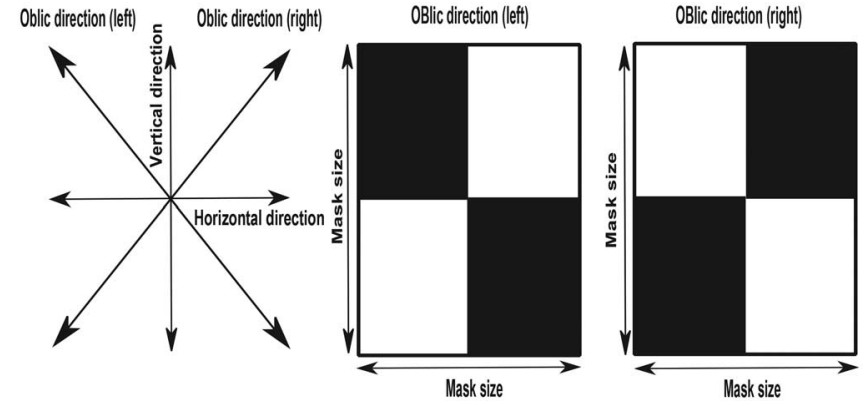

Fig. 4. Edge directions considered to calculate the ratio edge detector (left) and two masks used to calculate the ratio edge detector along the two oblique directions (right).

(e.g., the two black regions on Fig. 4), the ratio edge detector at neighborhood $n, R_{n}$ is calculated as follows:

$$
\begin{aligned}
X_{1} & =\frac{1}{N} \sum_{i=1}^{N} X_{i}, X_{2}=\frac{1}{N} \sum_{j=1}^{N} X_{j} \\
R_{n} & =X_{1} / X_{2}
\end{aligned}
$$

where $X_{i}$ and $X_{j}$ are the intensity values of the $i$ th pixel and $j$ th pixel for the patch $X_{1}$ and the patch $X_{2}$, respectively, and $N$ is the number of points (pixels) of each nonoverlapped region.

To detect most of the edges, the ratio edge detector must be applied in all the possible directions, i.e., the vertical and the horizontal directions and the two oblique directions (left and right) as shown in Fig. 4.

The ratio edge detector is calculated by applying a sliding mask to the ratio image centered at every pixel within the image. Two regions within this mask are split (for instance, in the oblique direction appearing in Fig. 4) and the ratio value for the related pixels in the two regions is computed. The ratio is computed for each direction and the minimum ratio (which is expected to be the most probable edge direction) is assigned to each point in the ratio image and then a binarized image is released. The binarized image is obtained after a classification step according to the following criteria: given two threshold values $T_{1} \leq 1$ and $T_{2} \geq 1$, the pixel $n$ under analysis is assigned to one of the two classes as follows:

1) edge class if $R_{n}<T_{1}$ or $R_{n}>T_{2}$;

2) homogenenous class in the other case.

The condition $T_{2}=1 / T_{1}$ holds to have a symmetrical operator and therefore, only one threshold value $T$ is used $\left(T=T_{1}\right)$. The $T$ control parameter depends on the number of looks of the image and it can be computed, assuming a gamma distribution, using the numerical scheme derived in [17]. However, for practical purposes, a suitable empirical value of $T$ for each mask size can be easily obtained after running the edge detector algorithm few times (usually two times) with variable $T$ values. For instance, given a mask of size $3 \times 3$, it is recommended to start the search for $T$ with a small $T$ value (around 0.1) and then, if no geometrical content is obtained, the threshold value is increased $(0.2,0.3, \ldots)$ till the structure is detected for this $T$ value. Then, for a larger mask, the search for a valid $T^{\prime}$ shall start at $T^{\prime}>T$, and the same approach is applied. 


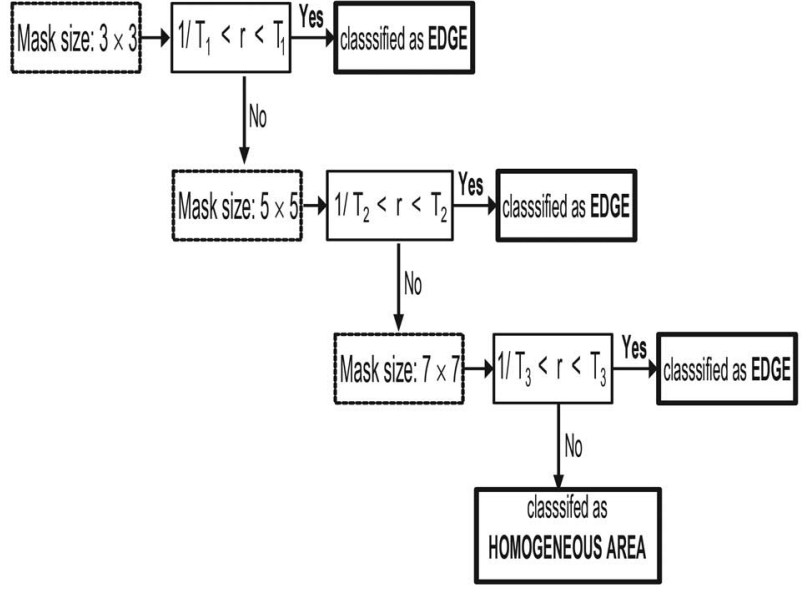

Fig. 5. Standard algorithm to estimate the ratio edge detector.

In order to efficiently detect the structure content within the image, this operation is performed several times using different window sizes to better capture all the details. Commonly, three estimations with window sizes of $3 \times 3,5 \times 5$, and $7 \times 7$ are applied. The value of the threshold $T$ increases with the window size (the larger the window size, the higher the threshold T). Fig. 5 illustrates the algorithm from [18] to estimate the edges by means of using three different mask sizes and the related threshold values. Note that small details (micro edges) are identified by using the small mask ( $3 \times 3$ in this case) and larger edges are captured by using larger mask sizes. The standard method includes an edge thinning (image morphological operation) to improve edge visualization.

The use of the standard algorithm is indicated to extract all possible edges within the image or for a particular filtering comparison of special difficulty. However, from a practical point of view of extracting relevant geometric content within the ratio image, the use of two masks of size $3 \times 3$ and $7 \times 7$ has been enough for all the experiments carried out in this proposal. Even for some cases, the use of a single mask has been enough. Note that by using a single mask, there is need of setting a unique threshold value $T$ too. The edge-thinning process has not been included in our implementation to better detect the remaining geometric content within the ratio image.

This detector is easy to compute and it demands only two control parameters: 1) mask size and 2) a threshold value $T$ to classify regions (homogeneous or a border).

A result using this edge estimator for the above ratio images (Fig. 2) is shown in Fig. 6, where it can be noted how edges, which were not visible in the ratio image, are now clearly marked in the binarized image.

Because speckle is a multiplicative noise, the usual estimation of gradient through a central difference scheme used in the standard Canny implementation does not seem appropriate to real SAR images [18]. This is the reason why detecting edges by applying the ratio edge detector is more satisfactory when dealing with SAR images. This becomes more efficient for the case of dealing with ratio images where using a ratio edge detector seems to be a natural approach.
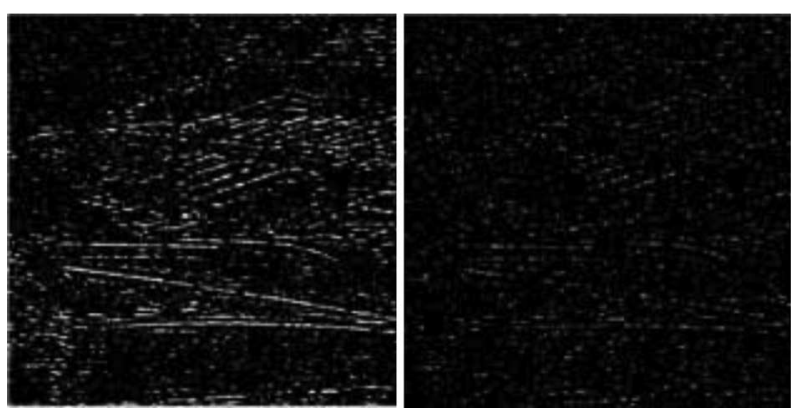

Fig. 6. Edges extracted by using the ratio edge detector for the ratio images for the Lee filter with mask size $7 \times 7$ (left) and the $\operatorname{SRAD}(t=15)$ filter (right).

\section{Adapting the $\beta$ Edge Estimator for SAR Ratio Images}

From above, it is clear that the geometric content remaining after the filtering operation can be indeed quantified from the edges extracted by applying the ratio edge detector. To do that, the standard $\beta$ edge estimator [8] is applied. This estimator is useful to compare edge preservation performance for filtered images and it is defined, for a given image $I$ in amplitude format and for the degraded filtered image $\hat{I}$ as

$$
\beta=\frac{\Gamma(\Delta I-\overline{\Delta I}, \widehat{\Delta I}-\widehat{\widehat{\Delta I}})}{\sqrt{\Gamma(\Delta I-\overline{\Delta I}, \Delta I-\overline{\Delta I})} \cdot \sqrt{\Gamma(\widehat{\Delta I}-\overline{\widehat{\Delta I}}, \widehat{\Delta I}-\widehat{\widehat{\Delta I}})}}
$$

where $\Gamma\left(I_{1}, I_{2}\right)$ is given by

$$
\Gamma\left(I_{1}, I_{2}\right)=\sum_{i=1}^{K} I_{1_{i}} \cdot I_{2_{i}} .
$$

$\Delta I$ and $\widehat{\Delta I}$ are the high-pass filtered version of the image $I$ and $\hat{I}$, respectively, obtained with a sliding Laplacian pixel kernel window of size $3 \times 3$ or another edge detector such as the Canny detector. $\overline{\Delta I}$ and $(\widehat{\widehat{\Delta I}})$ are the average value of the image $I$ and the average of the high-pass filtered version of the image $\widehat{\Delta I}$, respectively.

Note that this estimator evaluates the correlation between the ground-truth edges within the original image and the edges in the denoised image detected by means of the Laplacian filter (or the Canny filter). $\beta$ ranges between 0 and 1 , with unity for ideal edge preservation.

We adapt this definition of the $\beta$ estimator by replacing the image $I$ by the original (noisy) image, the degraded filtered image $\hat{I}$ by the ratio image $\Pi$, and the Canny edge detector by the ratio edge detector. This adapted $\beta$ estimator is renamed as $\beta_{\text {ratio }}$ estimator. From this definition of the $\beta_{\text {ratio }}$ estimator, a high value of the $\beta_{\text {ratio }}$ estimator means that some geometric content still appears in the ratio image.

To avoid spurious noise, the $\beta_{\text {ratio }}$ estimator has been modified to account only for geometric features of a minimum length (5-pixel length has been used for the experiments performed). To detect small edges (point targets or small details), this condition must be removed and spurious noise 
(isolated pixels) can be easily filtered out through basic image morphological operators.

\section{D. $\alpha \beta$-Ratio Estimator for Ratio Images}

As mentioned above, an ideal filter operation on an image implies that, in areas where speckle is fully developed, this ratio should have the features of pure speckle and no geometric content. The proposed referenceless estimator must take into account, on the first hand, the mean value of speckle and the $E N L$ measured in a homogeneous area within the ratio image, and on the other hand, the remaining geometrical content within the ratio image. Geometrical content is measured by means of the $\beta_{\text {ratio }}$ estimator explained above. The proposed estimator combines both measures to provide a score which can be used to rank a filtering operation. Therefore, we define the $\alpha \beta$-ratio estimator, $(\alpha \beta)$ as follows:

$$
\alpha \beta=\left\{\alpha \cdot\left|\delta_{E N L}\right|+(1-\alpha) \cdot\left|\delta_{\mu}\right|\right\}+\beta_{\text {ratio }}
$$

where $\alpha \in[0,1], \delta_{E N L}$ is the $E N L$ residue $\left(E N L_{n o i s y}-\right.$ $E N L_{\text {ratio }}$ ) and $\delta_{\mu}$ is the residue of the mean value of the speckle $\left(1-\mu_{\text {ratio }}\right)$. Both $E N L_{\text {ratio }}$ and $\mu_{\text {ratio }}$ values are measured in a homogeneous area in the ratio. $E N L_{n o i s y}$, if not known (it is generally known), may be estimated in a homogeneous ROI within the noisy image.

For an ideal filtering operation, $E N L_{\text {ratio }}$ would correspond to the original $E N L_{\text {noisy }}$ value of the original SAR image $\left(\delta_{E N L} \approx 0\right)$, and $\mu_{\text {ratio }}$ would be equal to $1\left(\delta_{\mu} \approx 0\right)$.

However, for a nonideal filter, it is expected that both $E N L_{\text {ratio }}$ and $\mu_{\text {ratio }}$ values would differ from the ideal ones $\left(E N L_{\text {ratio }}=E N L_{\text {noisy }}\right.$, and $\left.\mu_{\text {ratio }}=1\right)$. Therefore, the $\alpha$ value in the expression weighs, through a convex sum, the tradeoff for the $\delta_{E N L}$ and the $\delta_{\mu}$ values. That is, from a practical point of view, the user selects the $\alpha$ value according to his/her preferences, for instance, to weigh more the $\delta_{E N L}$ value against the preservation of the speckle mean value within the ratio image. Obviously, when comparing different despeckling filters, the $\alpha$ value must be the same for the sake of a fine comparison.

$\beta_{\text {ratio }}$ value is the one provided by the $\beta$ edge estimator. An ideal filter will be the one with $\alpha \beta=0$.

Finally, note that $\beta_{\text {ratio }}$ is adding (without a weight coefficient) to the terms dealing with the $E N L_{\text {ratio }}$, and $\mu_{\text {ratio }}$ values in (4). In this manner, the proposed $\alpha \beta$-ratio estimator strongly favors filters that preserve edges. However, for filters with similar $\beta_{\text {ratio }}$ values, the decision to rank the filters relies on the $\alpha, \delta_{E N L}$, and $\delta_{\mu}$ values.

Expression (4) must be evaluated in an homogeneous area selected within the ratio image. Indeed, as for an ideal filtering, the ratio image should contain only speckle, there is no need of choosing a homogeneous ROI to measure the $E N L_{\text {ratio }}$ and the $\mu_{\text {ratio }}$ (any area of the image could be selected). However, for practical purposes, to avoid problems with remaining content (mainly point targets), we recommend to use a homogeneous patch (the one selected for the noisy image to estimate the $\left.E N L_{n o i s y}\right)$. For a standard sensor and image postprocessing tasks, it is supposed that the whole noisy image shows a unique

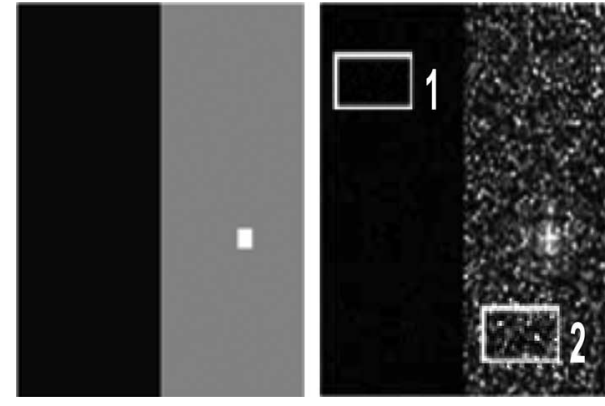

Fig. 7. (Left) Phantom with a bright scatter $(4 \times 4$ pixels $)$ and (right) image degraded with simulated speckle $(E N L=1)$. Homogeneous areas 1 (ROI-1) and 2 (ROI-2) to estimate $E N L_{\text {ratio }}$ and $\mu_{\text {ratio }}$ values are also represented.

$E N L_{n o i s y}$ value. So, just measuring $E N L_{\text {ratio }}$ and $\mu_{\text {ratio }}$ in a convenient ROI should be enough. Besides, $E N L_{n o i s y}$ is generally known and therefore, the homogeneous area must be only selected within the ratio image to estimate $E N L_{\text {ratio }}$ and the $\mu_{\text {ratio }}$ values.

Algorithm 1 describes the steps to calculate the $\alpha \beta$-ratio estimator using the ratio edge detector to extract the edges (geometric content) remaining within the ratio image. The program has been coded in MATLAB R2008a [19] and the computational cost for the phantom shown in this work (see Fig. 7) and applying one $7 \times 7$ mask is around $2 \mathrm{~s}$ in an Intel Core i7 Q7401.73-GHz machine.

\section{Algorithm 1. Computing the $\alpha \beta$-Ratio Estimator \\ input : \\ $\mathrm{U}$ : Corrupted (speckled) image \\ $\mathrm{V}$ : Denoised version of U \\ ROInoisy: Homogeneous patch for the noisy image ROIratio: Homogeneous patch for the ratio image ENLnoisy: ENL of the corrupted image muNoisy: Mean value of the ROI (corrupted image) $\mathrm{N}$ : Number of masks to calculate the edge ratio detector $S_{1}, \ldots, S_{N}$ : Size of the masks (edge ratio detector) $T_{1}, \ldots, T_{N}$ : Threshold values for the edge ratio detector alpha: weighting coefficient}

Output: The $\alpha \beta$-Ratio estimator: AlphaB

// Compute the ratio image $\Pi$

RatioImage $=\mathrm{U} / \mathrm{V}$;

$/ /$ Compute the $\beta_{\text {ratio }}$ edge estimator

EdgesCorruptedImage $=$ RatioEdgeDetector $(\mathrm{U}, \mathrm{N}, \mathrm{S}, \mathrm{T})$; EdgesRatioImage $=$ RatioEdgeDetector $($ RatioImage, N, S, T); BetaR = Beta(EdgesCorruptedImage, EdgesRatioImage $)$;

$/ /$ Estimate $\mu_{\text {ratio }}$ and $\sigma_{\text {ratio }}$

muRatio = Mean(ROIratio); // Mean ()$: \mu$ value

sigmaRatio = Sigma(ROIratio); // Sigma( $): \sigma$ value

// Estimate $E N L_{\text {ratio }}$

ENLratio $=$ muRatio $\cdot$ muRatio $/($ sigmaRatio $\cdot$ sigmaRatio $)$;

// Evaluate expression 4

$\mathrm{dENL}=\operatorname{abs}($ ENLnoisy - ENLratio); // abs: | $\mid$

dMSpeckle $=\operatorname{abs}(1-$ muRatio $) ; / /$ abs: $|\cdot|$

AlphaB $=($ alpha $\cdot$ dENL $+(1-$ alpha $) \cdot d$ MSpeckle $)+$ BetaR; 


\section{RESUlts}

The $\alpha \beta$-ratio estimator is intended for objectively evaluating performances of despeckling filters on real SAR images. However, an evaluation on a case with an available ground truth would be also of interest. Therefore, a set of experimental results has been obtained processing simulated SAR data and real SAR images.

For the simulated SAR image, a quantitative analysis is performed through well-established statistics estimators (mean and $E N L$ ), the FOM and the SSIM index estimators. The mean preservation and a high $E N L$ value measured within a region (or through the entire image) indicates a successful filter operation. The FOM estimator calculates an empirical distance between the ground-truth contours $I_{\text {ref }}$ and those obtained after the segmentation of the noisy image. As indicated in [20], this estimator is most frequently applied in image processing, although it has no theoretical proof. $F O M \in[0,1]$ with unity for ideal edge detection.

The SSIM index measures the similarity between the original and the despeckled image through a local statistical analysis of the image using the mean, the variance, and the covariance between the unfiltered and despeckled pixel values from the sliding window. SSIM $\in(-1,1)$ and a bad similarity between the original and the despeckled image corresponds to SSIM $\mapsto-1$, while a good similarity will be indicated by values SSIM $\mapsto+1$. We refer the reader to the references [20] and [21] for a complete description. The results include a visual comparison with other filtering strategies, such as the Lee filter and the SRAD filter.

\section{A. Results for an Image Corrupted With Speckle}

Fig. 7 shows the 1 -look $100 \times 100$ SAR phantom, the simulated image, and the two ROIs selected for evaluating the aforementioned quality estimators. The speckle has been simulated following the Gamma distribution with a mean value of 1 and fitted to provide an $E N L=1$. As it can be noted in the simulated image, a strong scatterer has been added to appreciate the effectiveness of the filters on preserving them. The phantom consists of two well different homogeneous areas sharing a nonabrupt edge.

The filtered image by the Lee filter $(7 \times 7$ mask size $)$ and the SRAD filter $(t=20)$ is shown in Fig. 8. The related ratio images and edges extracted by using the ratio edge detector $(7 \times 7$ mask with $T=0.4)$ are also shown in the same figure. It is observed that both filters perform well, preserving edges and notably reducing the image variance (this is more noticeable for the SRAD filter). Note also the preservation of the strong scatterer which is more evident for the case of applying the SRAD filter.

In the ratio images, it can be seen that filtering has slightly modified the edge boundary. This effect is more evident in the case of the SRAD filter, which, as expected, acts on the edge (this is known as the negative diffusion effect, which is particular of the SRAD filter). The Lee filter inhibits the filtering at the edge, but near to it, the filtering is less performed. Additionally, note that the strong scatterer is also seen in the ratio image. This clearly indicates that nonideal filtering has been done on it.
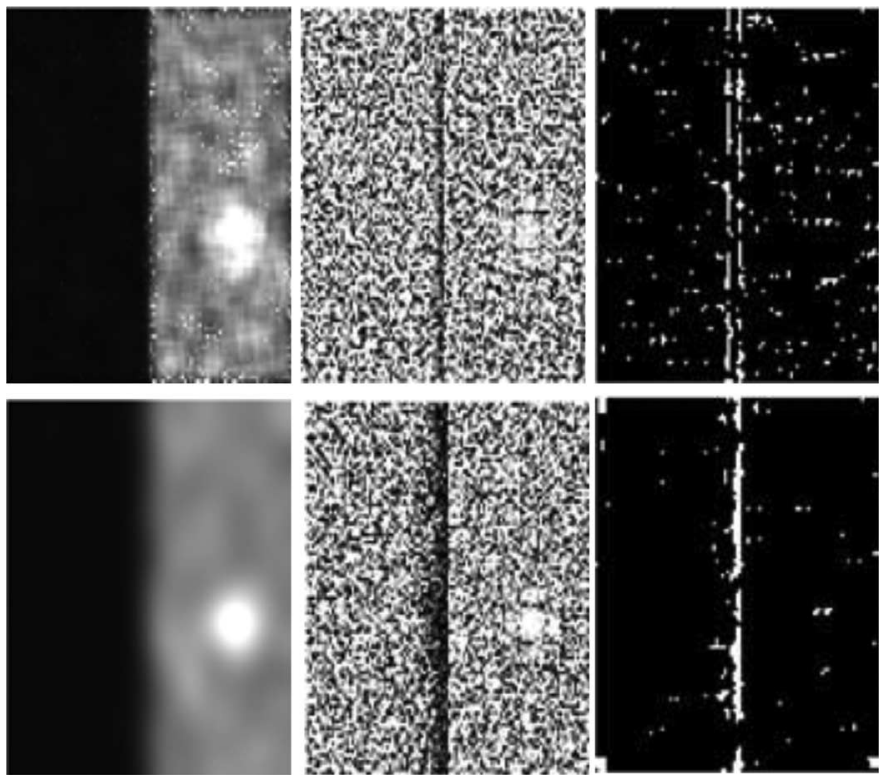

Fig. 8. Results for the SAR Phantom: (top row and left to right) The Lee filter $(7 \times 7)$, the ratio image, and the extracted edges from the ratio image. (Bottom row and left to right) The SRAD filter $(t=20)$, the ratio image, and the extracted edges from the ratio image.

Edges extracted by the edge ratio detector from the ratio images reveal that some geometric content still remains in the ratio image (just the edge or the edge boundaries). Note that to extract edges for the bright scatterer is a difficult task, but for both filters, some edges are slightly detected (more evident for the case of the SRAD filter).

Table II shows the quantitative performance evaluation for the filter realizations. As it can be seen, from the standard estimators evaluated (FOM, SSIM, ENL, and mean), it is not obvious to assess that one filter performs better than the other (look, for instance, the FOM and the SSIM values for both filters). However, for ratio ROI-1 and ratio ROI-2, the proposed $\alpha \beta$-ratio estimator indicates that, although more geometric content remains in the ratio image $\left(\beta_{\text {ratio }}=0.0762\right.$ for the SRAD filter against $\beta_{\text {ratio }}=0.0424$ for the Lee filter), the SRAD filter performs better than the Lee filter (ratio ROI-1: $\alpha \beta=$ 0.0948 against $\alpha \beta=0.1750$ for the Lee filter; ratio ROI-2: $\alpha \beta=0.0888$ against $\alpha \beta=0.1698$ for the Lee filter).

Note that the better $\alpha \beta$ value for the SRAD filter comes from the better values got at $\mu_{\text {ratio }}$ and $E N L_{\text {ratio }}$, whereas the value of the $\beta_{\text {ratio }}$ edge estimator is not excessively large compared to the $\beta_{\text {ratio }}$ value for the Lee filter.

\section{B. Testing the Consistency of the $\alpha \beta$-Ratio Estimator on Synthetic SAR Data}

The need for consistency for an image-quality index is one of the most well-established requirements. A natural manner to test the consistency of the proposed estimator consists of comparing its value for different results obtained for a given filter under different filter designs. It is expected that, for a bad filtering operation (for instance, an overfiltered result), $\alpha \beta$ will provide a high value. 
TABLE II

Quantitative Evaluation for the SAR Phantom (Best Values in Boldface)

\begin{tabular}{|c|c|c|c|c|c|c|c|}
\hline \multicolumn{2}{|l|}{ SAR Phantom } & Original & Simulated & Lee filter $(7 \times 7)$ & SRAD filter $(\boldsymbol{t}=20)$ & Lee filter $(21 \times 21)$ & SRAD filter $(\boldsymbol{t}=100)$ \\
\hline \multirow{2}{*}{ ROI-1 } & $\mu$ & 10 & 9.3943 & 9.4609 & 9.9966 & 9.6447 & 9.0805 \\
\hline & $E N L_{\text {noisy }}$ & -- & 1.0450 & 75.2548 & 434.4341 & 72.3664 & 48.0634 \\
\hline \multirow{2}{*}{ ROI-2 } & $\mu$ & 150 & 149.3808 & 150.3480 & 149.4753 & 154.1614 & 149.4347 \\
\hline & $E N L_{\text {noisy }}$ & -- & 1.0225 & 37.8220 & 488.9226 & 78.3991 & 25.4397 \\
\hline \multirow{2}{*}{ ROI-1, П image } & $\mu_{\text {ratio }}$ & - & 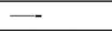 & 0.9698 & 1.0210 & 0.9713 & 1.0147 \\
\hline & ENL & $=$ & - & 1.2349 & 0.9838 & 0.3067 & 0.2547 \\
\hline \multirow{2}{*}{ ROI-2, П image } & $\mu_{\text {ratio }}$ & -- & - & 0.9631 & 0.9838 & 1.0206 & 1.0980 \\
\hline & ENL & - & - & 1.2179 & 1.0083 & 0.2928 & 0.2539 \\
\hline \multirow{2}{*}{ Whole image } & FOM & - & 0.1055 & 0.4505 & 0.4259 & 0.3453 & 0.0270 \\
\hline & SSIM & 二- & 0.2314 & 0.5836 & 0.7766 & 0.6687 & 0.5137 \\
\hline$\beta_{\text {ratio }}$ estimator & -- & - & 二- & 0.0424 & 0.0762 & 0.3684 & 0.4816 \\
\hline \multirow{2}{*}{$\alpha \beta$ estimator } & ROI-1 П & 一 & - & 0.1750 & 0.0948 & 0.7294 & 0.8616 \\
\hline & ROI-2 П & $=$ & $=$ & 0.1698 & 0.0888 & 0.7323 & 0.9037 \\
\hline
\end{tabular}

$\Pi$ Indicates Ratio Image and $\alpha=0.5$. Ideal Values are $\mu_{\text {ratio }}=1, E N L_{\text {ratio }}=1, \beta_{\text {ratio }}=0$, and $\alpha \beta=0$.
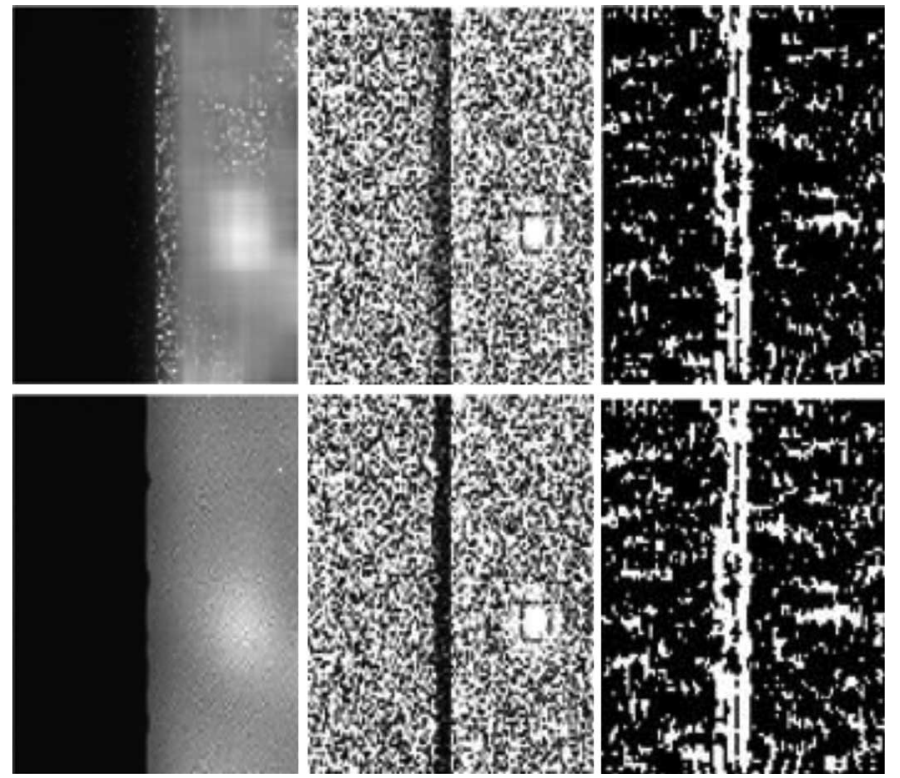

Fig. 9. Results for the SAR Phantom: (top row and left to right) The Lee filter $(21 \times 21)$, the ratio image, and the extracted edges from the ratio image. (Bottom row and left to right) The SRAD filter $(t=100)$, the ratio image, and the extracted edges from the ratio image.

In Fig. 9, some overfiltered results for the simulated SAR data obtained for the Lee filter (with a mask size $21 \times 21$ ) and for the SRAD filter $(t=100)$ are shown. The bright scatter appears much blurred and edges are clearly degraded for both filters (left and center images). The edges detected by the ratio edge detector (right image) are more visible (particularly for the SRAD filter). Numerical results for these images are collected in Table II (two last columns). As it can be seen, there is a correlation of $\beta_{\text {ratio }}$ values with the overall performances of the filters.

To finish this analysis, note that the $\alpha \beta$-ratio estimator gets similar values for the two ROIs selected in the $\Pi$ image. This confirms the consistency of the proposed estimator and it also means that it suffices to select a unique homogeneous ROI to correctly evaluate $\alpha \beta$.

Besides, the FOM values (which accounts for edge preservation), measured as usual, i.e., on the filtered images, agree with the values obtained by the $\alpha \beta$-ratio estimator. That is, high
FOM values correspond to low $\alpha \beta$-ratio values and the opposite also holds true. This also confirms the consistency of the proposed estimator with a standard edge-based estimator.

\section{Real Images Results}

To evaluate the performances of the new estimator on real SAR images, four despeckling filters have been selected: the Lee filter, the SRAD filter, the Bayesian nonlocal (BNL) filter, and the enhanced Bayesian nonlocal (EBNL) filter. The real SAR image selected is the one shown in Fig. 1 (top left) and visual results for the Lee filter and the SRAD filter are also shown in this figure (top right for the Lee filter, and bottom left for the SRAD filter with $t=15$ ) and not repeated in this section.

The BNL-means filter [22] aims to reduce the Bayesian risk by estimating the prior probability on the noisy image patch. Bayesian-like filters are recognized as excellent filters in terms of mean, edge, and detail preservation. The EBNL filter [6] is an improved version of the BNL filter which combines the sigma preselection by Lee [3] and a procedure to filter dark areas, which provides filtered images of great contrast.

Visual results for the EBNL, the BNL, the Lee. and the SRAD filters, as it can be seen in Fig. 10, and Fig. 1, are notably different: the EBNL filter performs better than the other three filters on preserving the bright scatterers due to the sigma preselection approach (notice, e.g., the evident differences for the bright targets in this EBNL and BNL filtered images). Besides, the EBNL image shows a greater contrast, and homogeneous dark areas are specially well filtered (the EBNL filtered image reveals a remarkable contrast compared to the results obtained by the BNL, the Lee, and the SRAD filters). See how the EBNL filter performs on dark areas in [6].

The amount of geometric content visible in the ratio images for both Bayesian filters are similar and comparable to the one provided by the SRAD filter (see Fig. 6) and minor than the geometric content provided by the Lee filter.

In Table III, the values of the $\mu_{\text {ratio }}$, the $E N L_{\text {ratio }}$, the $\beta_{\text {ratio }}$ edge estimator, and the $\alpha \beta$ estimators are collected. For this SAR image, $E N L_{\text {ratio }}=1$ and the value of the speckle mean $\mu_{\text {ratio }}$ should be equal to $1 . \alpha \beta=0.4810$ for the Lee filter (last position in the ranking among the visually shown 

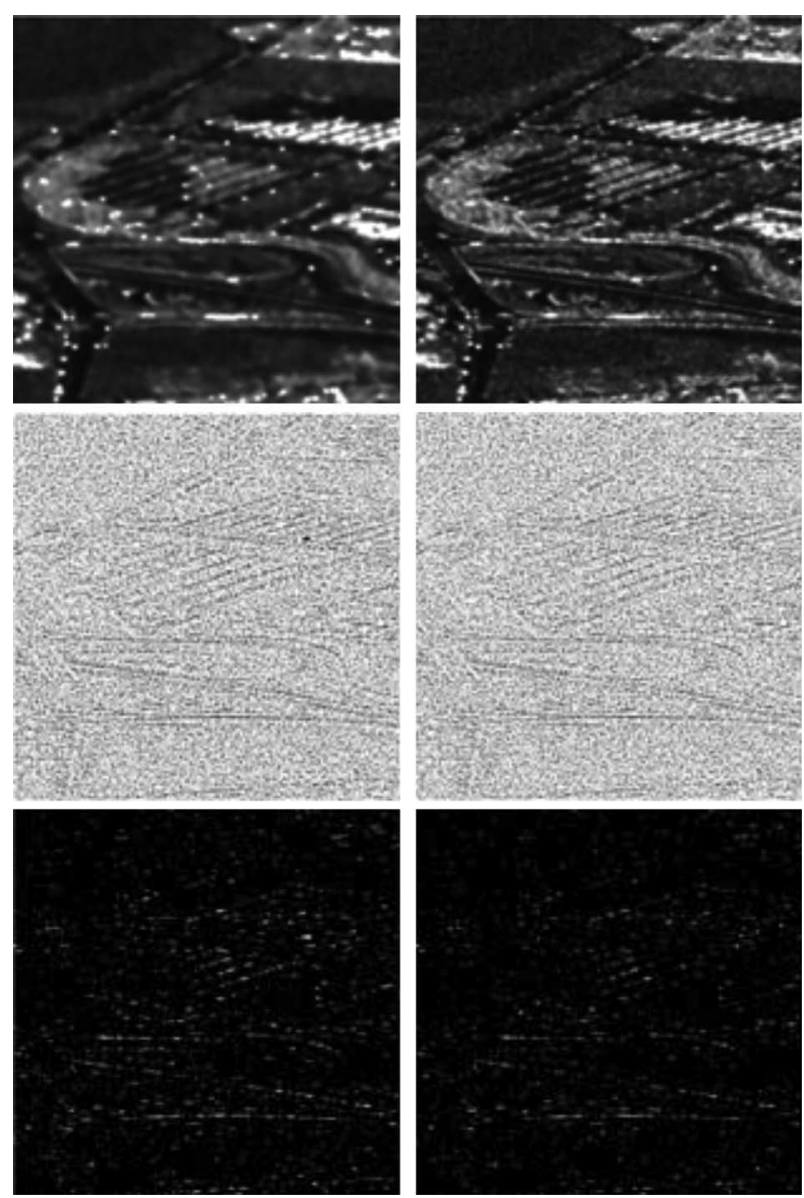

Fig. 10. Comparison of filtering operation on original SAR image: (top left) the EBNL filter (search window: $11 \times 11$, patch: $5 \times 5$, iterations: 3 ), the ratio image (middle left), and the corresponding edges detected using the ratio edge estimator (bottom left). (Top right) The BNL filter (search window: $11 \times 11$, patch: $5 \times 5$, iterations: 3 ), the ratio image (middle right), and the corresponding edges detected using the ratio edge estimator (bottom right).

TABLE III

QUANTITATIVE EVALUATION FOR THE REAL SAR IMAGE (Best Values in Boldface)

\begin{tabular}{|l||l|l|l|l|}
\hline Filter $($ ranked according to $\alpha \beta)$ & $\mu_{\text {ratio }}$ & $E N L_{\text {ratio }}$ & $\beta_{\text {ratio }}$ & $\alpha \beta$ \\
\hline \hline$\left(1^{\circ}\right)$ SRAD filter $(t=15)$ & 0.9926 & 1.0645 & 0.2881 & $\mathbf{0 . 3 2 4 1}$ \\
\hline$\left(2^{\circ}\right)$ EBNL filter $(11 \times 11,3 \times 3)$ & 0.9709 & 1.2162 & $\mathbf{0 . 2 3 4 5}$ & 0.3571 \\
\hline$\left(3^{\circ}\right)$ BNL filter $(11 \times 11,3 \times 3)$ & 0.9737 & 1.1879 & 0.2829 & 0.3900 \\
\hline$\left(4^{\circ}\right)$ Lee filter $(7 \times 7)$ & $\mathbf{0 . 9 9 9 8}$ & $\mathbf{1 . 0 4 6 9}$ & 0.4575 & 0.4810 \\
\hline \hline$\left(5^{\circ}\right)$ Lee filter $(15 \times 15)$ & 1.0104 & 0.9191 & 0.7604 & 0.8064 \\
\hline$\left(6^{\circ}\right)$ SRAD filter $(t=100)$ & 1.0247 & 0.9145 & 0.8029 & 0.8580 \\
\hline
\end{tabular}

$\alpha=0.5$. Ideal Values are $\mu_{\text {ratio }}=1, E N L_{\text {ratio }}=1, \beta_{\text {ratio }}=0$, and $\alpha \beta=0$.

results, according to the proposed ratio estimator) and $\alpha \beta=$ 0.3241 for the SRAD filter. These values for the $\alpha \beta$ estimators correspond to $\alpha=1$. However, by setting $\alpha=0.1$, to evaluate the performances of the filters assigning more weight to the preservation of the $\mu_{\text {ratio }}$ than to the preservation of $E N L_{\text {ratio }}$, the best filter is the EBNL ( $\alpha \beta=0.2823$ against $\alpha \beta=0.3012$ for the SRAD filter).

Note that the SRAD filter performed much better than the Lee filter for the phantom corrupted with speckle and also for this case (for both selected $\alpha$ values). The superior performance of the SRAD filter in comparison to the enhanced Lee filtered
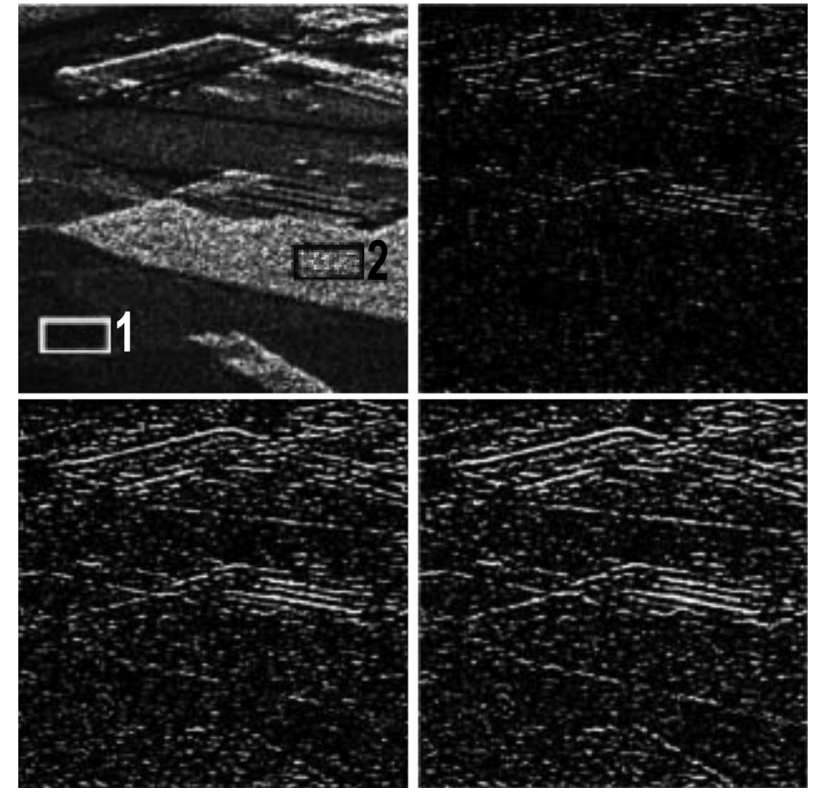

Fig. 11. Results for the consistency test for the real SAR image: (top left) Real SAR image. (Top right) extracted edges by the ratio edge detector for the Lee filter $(7 \times 7)$. (Bottom left) extracted edges by the ratio edge detector for the Lee filter $(11 \times 11)$. (Bottom right) extracted edges by the ratio edge detector for the Lee filter $(15 \times 15)$. Homogeneous areas $1($ ROI-1) and 2 (ROI-2) to estimate $E N L_{\text {ratio }}$ and $\mu_{\text {ratio }}$ values are also represented.

is analyzed in [4]. Because the filter applied is the original Lee filter (and not its enhanced version), the comparison favors even more the result for the SRAD filter.

It is interesting to observe that, visually, the EBNL filter seems better than the SRAD filter, but this is a subjective evaluation. Numerical data confirm that, according to the proposed ratio estimator, for this image and for the filters applied (each filter with the indicated design), the SRAD filter gets the first position in the ranking $(\alpha=1)$ as the best filter, combining an excellent edge preservation with $E N L_{\text {ratio }}$ and $\mu_{\text {ratio }}$ values.

The results in Table III justifies the need of the $\alpha \beta$ estimator, because, from the the visual inspection of the ratio images, it is hard to rank, for this SAR image, the performances of the SRAD, the EBNL, the BNL, and the Lee filters.

We remark that we tested the new proposed estimator on nonpolarimetric SAR data. A discussion of the influence of polarization, radar frequency, bandwidth, and radar geometry would require a general discussion of the effects of these parameters on data coming from a wide variety of targets [23]. We think that this is far away from the scope of this work. Similar analysis to the one performed above must be carried out to assess whether the proposed estimator can be applied to polarimetric SAR data to rank despeckling filters.

\section{Testing the Consistency of the $\alpha \beta$-Ratio Estimator on Real SAR Data}

In Table III, the overfiltered solutions obtained by the Lee filter $(15 \times 15)$ and the SRAD filter $(t=100)$ for the real SAR image shown in Fig. 1 are collected. As it can be seen, the results obtained are ranked in the last positions. 
TABLE IV

Quantitative Analysis of THE CONSISTENCy Test For THE REAL SAR IMAGE (BEST VALUES IN BoldFACE)

\begin{tabular}{|l||l||l|l|l|}
\hline \multicolumn{2}{|l|}{ Filter } & $\mu_{\text {ratio }}$ & $E N L_{\text {ratio }}$ & $\alpha \beta$ \\
\hline \hline \multirow{2}{*}{ The Lee filter $(7 \times 7), \beta_{\text {ratio }}=0.3502$} & ROI-1 & 0.9798 & 0.9305 & 0.3951 \\
\cline { 2 - 5 } & ROI-2 & 0.9978 & 1.3079 & 0.5052 \\
\hline \multirow{2}{*}{ The Lee filter $(11 \times 11), \beta_{\text {ratio }}=0.5770$} & ROI-1 & 0.9657 & 0.8738 & 0.6572 \\
\cline { 2 - 5 } & ROI-2 & 1.0090 & 1.1062 & 0.6346 \\
\hline \multirow{2}{*}{ The Lee filter $(15 \times 15), \beta_{\text {ratio }}=0.6418$} & ROI-1 & 0.9374 & 0.8212 & 0.7625 \\
\cline { 2 - 5 } & ROI-2 & 1.0137 & $\mathbf{1 . 0 3 0 4}$ & 0.6639 \\
\hline \hline \multirow{2}{*}{ The SRAD filter $(t=10), \beta_{\text {ratio }}=\mathbf{0 . 2 4 9 7}$} & ROI-1 & 0.9706 & 0.9653 & $\mathbf{0 . 2 8 1 7}$ \\
\cline { 2 - 5 } & ROI-2 & $\mathbf{1 . 0 0 6 3}$ & 1.2667 & 0.3862 \\
\hline \multirow{2}{*}{ The SRAD filter $(t=20), \beta_{\text {ratio }}=0.4642$} & ROI-1 & 0.9556 & 0.8853 & 0.5437 \\
\cline { 2 - 5 } & ROI-2 & 1.0119 & 1.1046 & 0.5225 \\
\hline \multirow{2}{*}{ The SRAD filter $(t=50), \beta_{\text {ratio }}=0.5999$} & ROI-1 & 0.9143 & 0.8330 & 0.7263 \\
\cline { 2 - 5 } & ROI-2 & 1.0123 & 1.0363 & 0.6242 \\
\hline
\end{tabular}

$\alpha=0.5$. Ideal Values are $\mu_{\text {ratio }}=1, E N L_{\text {ratio }}=1, \beta_{\text {ratio }}=0$, and $\alpha \beta=0$.

We complete the consistency test by discussing some results for another real SAR data. A different $(500 \times 500)$ ROI from the same 1-look HH SAR (Oberpfaffenhofen, Germany) was chosen (Fig. 11, top left) and filtered by the Lee filter and the SRAD filter. Two homogeneous areas have been selected (ROI1 and ROI-2) to evaluate the $\alpha \beta$-ratio estimator and to check the possible dependency of its value with the chosen ROIs. In the same figure, only the results (edges extracted within the ratio images $)$ for the Lee filter with different mask sizes $(7 \times 7$, $11 \times 11$, and $15 \times 15)$ are shown. The increase in the geometric content with the increasing size of the mask applied is quite noticeable. As a consequence of that, $\beta_{\text {ratio }}$ values must also increase.

The numerical values related to the estimators, for the Lee and the SRAD filters, are collected in Table IV. It is important to note how the $\alpha \beta$ estimator may be correctly evaluated from and unique ROI from the ratio image. This result greatly simplifies the computation of the $\alpha \beta$ estimator.

Once again, the results were consistent and they respond to the expected one for the proposed estimator.

\section{CONCLUSION}

Comparing different filter performances is an intricate task even through the existing plethora of image quality indexes. A new referenceless image-quality index based on the ratio edge detector to help in evaluating a filter realization on real SAR images has been presented. The need of such estimator has been extensively discussed and justified using for that purpose several efficient despeckling filters. Some results for a synthetic image corrupted with one look speckle, and for real one look SAR images clearly shown that the new $\alpha \beta$-ratio estimator certainly allows to objectively evaluating more in detail the performances of a filtering operation on an SAR image.

The proposed $\alpha \beta$-ratio estimator is easy to compute and intuitively simple. A relevant characteristic is that it favors filters that preserve edges, which is of especial relevance in the context of SAR imagery.

The consistency of the proposed estimator has been tested under several experiments with synthetic data and real SAR images. For all cases, the results shown that for bad filtering designs, $\alpha \beta$ gets high values and that it is specially sensible to degradation of edges after filtering operations.
The difference of this new estimator with existing imagequality indexes is that the remaining geometric content within the ratio image is taken into account. Besides, the new estimator is the first one evaluated within the ratio image.

Future work includes extending this study to compare performances of other competitive despeckling filters (for instance, total variation-based filters), and to enhance the $\alpha \beta$-ratio estimator to account for the remaining geometric content related to small bright scatterers. Rigorous analysis of how to select the optimal threshold values and the corresponding mask sizes used by the ratio edge detector is required for detecting small remaining features within the ratio images.

Additionally, this work may be extended to deal with polarimetric SAR data.

\section{REFERENCES}

[1] V. S. Frost et al., "A model for radar images and its application to adaptive digital filtering of multiplicative noise," IEEE Trans. Pattern Anal. Mach. Intell., vol. PAMI-4, no. 2, pp. 157-165, Mar. 1982.

[2] I. Duskunovic et al., "Urban area detection in SAR imagery using a new speckle reduction technique and Markov random field texture classification," in Proc. Geosci. Remote Sens. Symp. (IGARSS'00), 2000, vol. 2, pp. $636-638$.

[3] J. S. Lee et al., "Improved sigma filter for speckle filtering of SAR imagery," IEEE Trans. Geosci. Remote Sens., vol. 47, no. 1, pp. 202-213, Jan. 2009

[4] Y. Yu and S. T. Acton, "Speckle reducing anisotropic diffusion," IEEE Trans. Image Process., vol. 11, no. 11, pp. 1260-1270, Nov. 2002.

[5] A. Buades, B. Coll, and J. M. Morel, "A review of image denoising algorithms with a new one," SIAM Interdiscip. J. Multiscale Model. Simul., vol. 4, no. 2, pp. 490-530, 2005.

[6] H. Zhong, Y. Li, and L. Jiao, "SAR image despeckling using Bayesian nonlocal means filter with sigma preselection," IEEE Geosci. Remote Sens. Lett., vol. 8, no. 4, pp. 809-813, Jul. 2011.

[7] L. Gomez et al., "Supervised constrained optimization of Bayesian nonlocal means filter with sigma preselection for despeckling SAR images," IEEE Trans. Geosci. Remote Sens., vol. 51, no. 8, pp. 4563-4575, Aug. 2013

[8] A. Achim, E. Kuruoglu, and J. Zerubia, "SAR image filtering based on the heavy-tailed Rayleigh model," Inst. Nat. Recherche Informat. Automatique (INRIA), France, Tech. Rep. 5493, 2005.

[9] O. Bustos, D. Flessia, and A. Frery, "Simulation of correlated intensity SAR images," in Proc. XII Braz. Symp. Comput. Graph. Image Process., 1999, pp. 317-326.

[10] W. K. Pratt, Digital Image Processing. Hoboken, NJ, USA: Wiley, 1977.

[11] Z. Wang et al., "Image quality assessment: From error visibility to structural similarity," IEEE Trans. Image Process., vol. 13, no. 4, pp. 600-612, Apr. 2004.

[12] A. Mittal, A. K. Moorthy, and A. C. Bovik, "No-reference image quality assessment in the spatial domain," IEEE Trans. Image Process., vol. 21, no. 12 , pp. 4695-4708, Dec. 2012.

[13] S. Parrilli, M. Poderico, C. V. Angelino, and L. Verdoliva, "A nonlocal SAR image denoising algorithm based on LLMMSE wavelet shrinkage," IEEE Trans. Geosci. Remote Sens., vol. 50, no. 2, pp. 606-616, Feb. 2012.

[14] J. S. Lee, "Digital image enhancement and noise filtering by using local statistics," IEEE Trans. Pattern Anal. Mach. Intell., vol. PAM1-2, no. 2, pp. 165-168, Mar. 1980.

[15] P. Perona and J. Malik, "Scale space and edge detection using anisotropic diffusion," IEEE Trans. Pattern Anal. Mach. Intell., vol. 12, no. 7, pp. 629-639, Jul. 1990.

[16] J. Canny, "A computational approach to edge detection," IEEE Trans. Pattern Anal. Mach. Intell., vol. PAMI-8, no. 6, pp. 679-698, Nov. 1986.

[17] R. Touzi, A. Lopes, and P. Bousquet, "A statistical and geometrical edge detector for SAR images," IEEE Trans. Image Process., vol. 26, no. 6, pp. 764-773, Nov. 1998.

[18] Y. Desnos and V. Matteini, "Review on structure detection and speckle filtering on ERS-1 images," EARSeL Adv. Remote Sens., vol. 2, no. 2, pp. 52-65, 1993.

[19] Matlab, The MathWorks, 2009. 
[20] S. Chabrier, H. Lauren, C. Rosenberger, and B. Emile, "Comparative study of contour detection evaluation criteria based on dissimilarity measures," J. Image Video Process., vol. 2008, no. 2, pp. 1-14, 2008.

[21] Z. Wang, A. Bovik, H. Sheikh, and E. Simoncelli, "Image quality assessment: From error visibility to structural similarity," IEEE Trans. Image Process., vol. 13, no. 4, pp. 600-611, Apr. 2004

[22] H. Zhong, Y. Li, and L. Jiao, "Bayesian nonlocal means filter for SAR despeckling," in Proc. Asia Pac. Conf. Synth. Aperture Radar, Xian, China, 2009, pp. 1096-1099.

[23] C. López-Martínez, "Polarimetric SAR speckle noise model," IEEE Trans. Geosci. Remote Sens., vol. 41, no. 10, pp. 2232-2242, Oct. 2003.

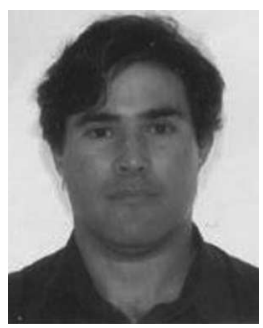

Luis Gomez (M'00) received the Ph.D. degree in telecommunication engineering from the University of Las Palmas de Gran Canaria, Las Palmas de Gran Canaria, Spain.

Since 1994, he has been an Associate Professor with the Department of Electronic Engineering and Automatic (DIEA), University of Las Palmas de Gran Canaria. His research interests include image processing (computer vision), remote sensing for SAR, and polarimetric SAR (PolSAR).

Dr. Gomez is currently an Associate Editor of the IEEE Geoscience and Remote Sensing Letters and belongs to the Editorial Board of Image Processing on Line (IPOL; www.ipol.im).

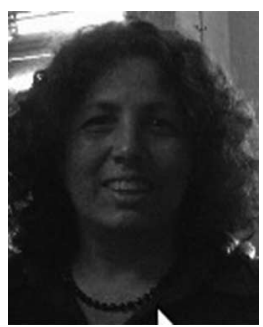

Mara Elena Buemi received the degree in computer science and the Ph.D. degree in computer science from the University of Buenos Aires, Buenos Aires, Argentina, in 2003 and 2012, respectively.

Currently, she is a JTP (Jefe de Trabajos Prácticos, Laboratory Assistant Professor) with the Computer Science Department, Faculty of Exact and Natural Sciences, University of Buenos Aires. Her research interests include image processing, remote sensing, and computer vision.

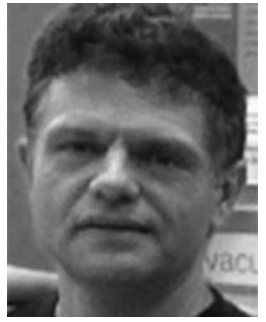

Julio C. Jacobo-Berlles received the Eng. degree in electronics and the Ph.D. degree in computer science from the University of Buenos Aires, Buenos Aires, Argentina, in 1983 and 2005, respectively.

currently, he is an Adjoint Professor with the Computer Science Department, Faculty of Exact and Natural Sciences, University of Buenos Aires. His research interests include microwave remote sensing, image processing, and computer vision.

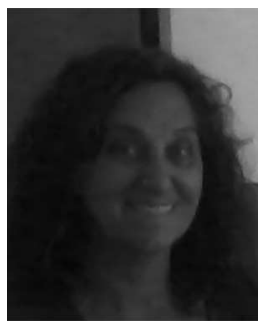

Marta E. Mejail received the Master's degree in mathematics and the Ph.D. degree in computer science from the University of Buenos Aires, Buenos Aires, Argentina, 1980 and 1999, respectively.

Currently, she is a Professor and the Head of the Computer Vision and Image Processing Group, Computer Science Department, Faculty of Exact and Natural Sciences, University of Buenos Aires. 